\title{
Entrapment of guide wire by Chiari's network during pacemaker implantation
}

\author{
Weiliang Zhu ${ }^{1}$, Zhao Jin ${ }^{2}$, Qiang $\mathrm{Li}^{1}$ \\ ${ }^{1}$ Department of Cardiology, Xiamen Cardiovascular Hospital, Xiamen University, Xiamen, China \\ ${ }^{2}$ School of Medicine, Xiamen University, Xiamen, China
}

A 35-year-old female patient was diagnosed with sick sinus syndrome and needed a pacemaker implantation. During the operation, the left subclavian vein was punctured when a J wire was attempting to be placed into the inferior vena cava to ensure entry to the venous system. In the process of adjusting position of the $J$ wire, it was found that the $\mathrm{J}$ wire was entrapped in the junctional area of the lower right atrium and inferior vena cava. The $\mathrm{J}$ wire was rotated and was attempted to be pulled out, but it failed. Finally, the wire was forcefully pulled out, and it was found that the tip of J wire was wrapped completely around Chiari's network (Fig. 1).

Although it has never been reported that the wire was entrapped in Chiari's network was pulled out by force, it is not encouraged in consideration of the high risk of tearing the inferior vena cava. With the development of devices, new choices will be available to deal with this problem. It has been reported that an entrapped pacing lead was pulled out successfully with the use of a laser sheath and intracardiac echocardiogram. This method should be considered a good choice with less risk of injury. Above all, during cardiac intervention, rotating the $\mathrm{J}$ wire, lead or catheter in the junctional area of the lower right atrium and inferior vena cava should be avoided.

Funding: This work was supported by research grant No. $3502 Z 20174005$ from Huimin Project of Xiamen Science and Technology Bureau.

Conflict of interest: None declared

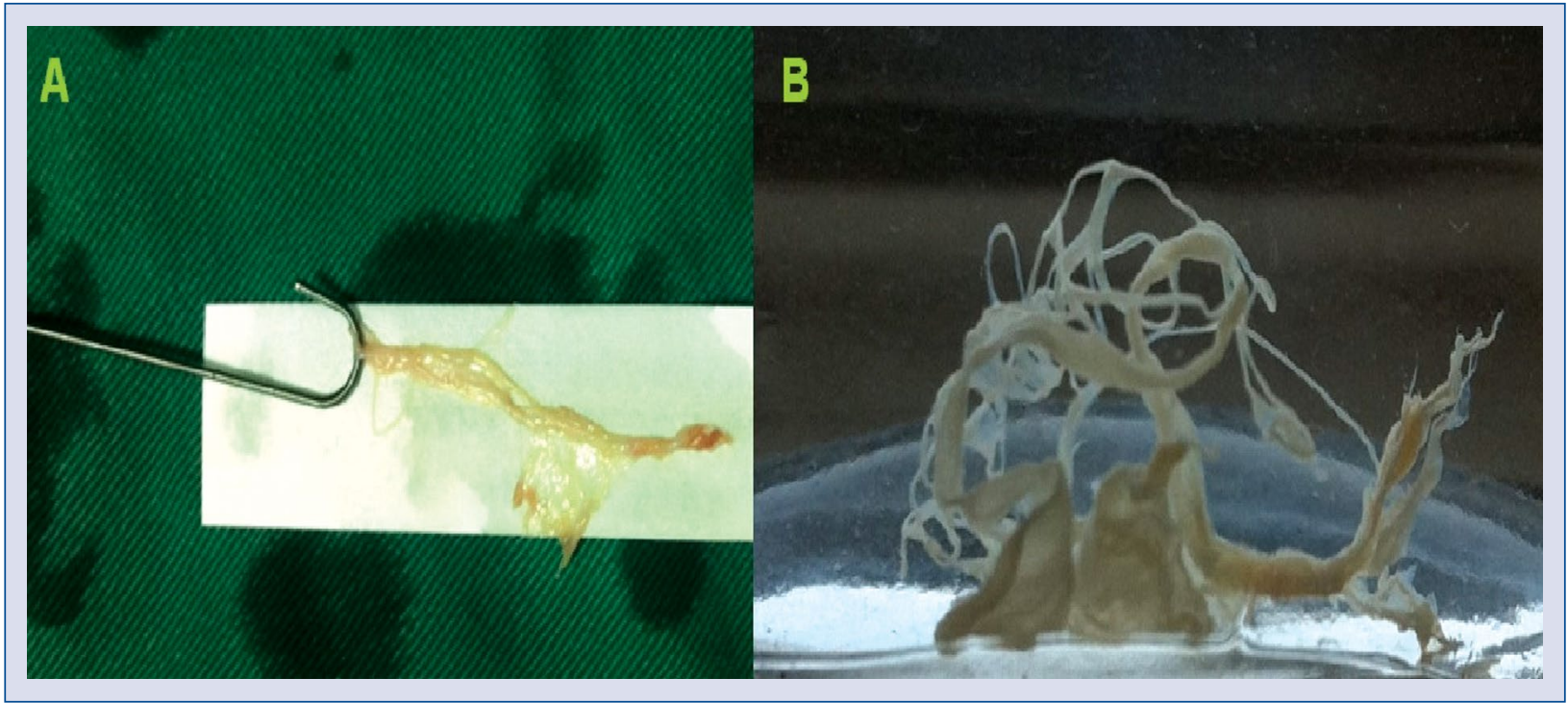

Figure 1. A. Chiari's network wrapped around the tip of $\mathrm{J}$ wire; B. Complete Chiari's network was shown in the water.

Address for correspondence: Dr. Qiang Li, 205 Hubin South Road, Xiamen, Fujian 361000, China, tel: +86 15359293670, e-mail: liqiang@xmu.edu.cn

Received: 24.05.20199 Accepted: 23.06.2019 